\title{
Assessing Pancreatic Cancer Risk Associated with Dipeptidyl Peptidase 4 Inhibitors: Data Mining of FDA Adverse Event Reporting System (FAERS)
}

Xiaodong Feng ${ }^{1 *}$, Amie Cai ${ }^{3}$, Kevin Dong ${ }^{1}$, Wendy Chaing ${ }^{1}$, Max Feng ${ }^{4}$, Nilesh S Bhutada ${ }^{1}$, John Inciardi ${ }^{1}$ and Tibebe Woldemariam ${ }^{2}$

${ }^{1}$ Department of Clinical and Administrative Sciences, California Northstate University College of Pharmacy, Rancho Cordova, CA, USA

${ }^{2}$ Department of Pharmaceutical and Biomedical Sciences, California Northstate University College of Pharmacy, Rancho Cordova, CA, USA

${ }^{3}$ Department of Molecular \& Cell Biology, University of Berkeley, Berkeley, CA, USA

${ }^{4}$ Rio Americano High School, Carmichael, CA, USA

\begin{abstract}
Background: Identifying cancer risks associated with medicinal agents plays an important role in cancer control and prevention. Case reports of cancers associated with pharmacotherapy have been escalating in the Food and Drug Administration Adverse Event Reporting System (FAERS). The objective of this study is to assess the risk of pancreatic cancer associated with anti-diabetic drugs of dipeptidyl peptidase 4 (DPP 4) inhibitors with or without combination of metformin.
\end{abstract}

Methods: Using the FAERS public database, the adverse event reports (ADRs) associated with widely used DPP 4 inhibitors with or without combination of metformin were generated and evaluated. Standardized pharmacovigilance tools were applied to detect the signal for cancer risks by calculating the proportional reporting ratio (PRR) and the reporting odds ratio (ROR).

Results: Among 12618 ADRs associated with sitagliptin from 2007 to 2011, there were 223 cases of cancer. There was a significant correlation between the cancer reporting ratio and the time $(R=0.796, P<0.001)$. Pancreatic cancers accounted for $22 \%$ of all combined cancer adverse events. Pharmacovigilance assessment from 2007 to 2012 indicated that there was a significant risk of pancreatic cancer associated with DPP 4 inhibitors treatment (ROR=5.922). Interestingly, minimal risk of pancreatic cancer risk was associated with metformin (ROR=1.214). Combination of DPP 4 inhibitor sitagliptin with metformin correlates with significantly lower risk of pancreatic cancer compared to sitagliptin treatment without metformin (OR=0.277, 95\% Cl: $0.210-0.365)$.

Interpretation: There was a significant signal of pancreatic cancer risk associated with DPP 4 inhibitor treatment. For the first time we demonstrated that combination with metformin significantly reduced the risk signal of pancreatic cancer associated with DPP 4 inhibitors in FAERS. Considering the limitation of the FAERS, this study implied the potential strategy for cancer control and prevention in diabetic patients, and provided directions for future clinical studies.

Keywords: Dipeptidyl peptidase 4; Metformin; Food and Drug Administration (FDA)

\section{Introduction}

Today's media landscape is constantly evolving toward more efficient tools of communication that can gather unprecedented amounts of information. This new media landscape offers tremendous opportunity for the public, health care professionals, pharmaceutical companies, biomedical researchers, and public health agencies to share, discuss, inquire, and report health care information using traditional and innovative media tools [1-3]. The new social media landscape also encourages health care professionals and pharmaceutical companies to face the dilemma of engaging consumers without the fear of liability when clarifying, monitoring and reporting the adverse events [4]. This dilemma creates an important role for the government to step in and engage all the stakeholders of public health and proactively monitor and regulate the adverse events. The public databases hosted by the federal government serve as important information hubs to collect, evaluate and distribute health care information, which plays important role in diseases control and prevention in the changing media landscape and new information ecology [5].

The Food and Drug Administration Adverse Event Reporting System (FAERS) represents one of the most successful digital tools of media landscape sponsored by the United States government. The FAERS is a public media platform for consumers and clinicians to address their concerns of drug safety by reporting the adverse drug events to the Food and Drug Administration (FDA) [6]. The FAERS creates new information ecology by providing an open communication channel between consumers, clinicians and the FDA [7]. It also has the potential to serve as a whistleblower by sending alerts and alarms of drug safety signals, such as cancer risks associated with drug treatment, to the federal agencies, pharmaceutical companies, health care professionals, consumers and drug safety advocates [8]. In addition, clinical research based on the data from the FAERS can suggest future directions for pharmaceutical companies and clinical research institutions when designing studies to address drug safety issues.

Along with environmental and genetic risk factors, drugs play an important role in cancer development. Identifying cancer events associated with specific drugs offers an essential solution for cancer control and prevention $[9,10]$. Every year several hundred thousand of drug related cancer events are communicated by health care consumers

*Corresponding author: Xiaodong Feng, PharmD, PhD, Department of Clinical and Administrative sciences, California Northstate University College of Pharmacy, Rancho Cordova, CA95670, USA, Tel: 916-503-1812; Fax: 916-631-8127; E-mail: xfeng@cnsu.edu

Received March 25, 2013; Accepted July 23, 2013; Published July 25, 2013

Citation: Feng X, Cai A, Dong K, Chaing W, Feng M, et al. (2013) Assessing Pancreatic Cancer Risk Associated with Dipeptidyl Peptidase 4 Inhibitors: Data Mining of FDA Adverse Event Reporting System (FAERS). J Pharmacovigilance 1: 110. doi:10.4172/2329-6887.1000110

Copyright: $\odot 2013$ Feng X, et al. This is an open-access article distributed under the terms of the Creative Commons Attribution License, which permits unrestricted use, distribution, and reproduction in any medium, provided the original author and source are credited. 
Citation: Feng X, Cai A, Dong K, Chaing W, Feng M, et al. (2013) Assessing Pancreatic Cancer Risk Associated with Dipeptidyl Peptidase 4 Inhibitors: Data Mining of FDA Adverse Event Reporting System (FAERS). J Pharmacovigilance 1: 110. doi:10.4172/2329-6887.1000110

to their health care providers, and most of these adverse events are reported to the FDA through the digital media of FAERS [6].

Dipeptidyl peptidase 4 (DPP 4) is widely expressed in human epithelial cells, microvascular endothelial cells and retinal cells. It serves multiple functions in cleaving a variety of physiologically important peptides in the circulation, such as certain chemokines, mitogenic growth factors and incretin hormones, including glucagon-like peptide-1 (GLP-1) and glucose-dependent insulinotropic polypeptide (GIP). With sitagliptin, saxagliptin and linagliptin approval in the United States, DPP 4 has been a successful drug target for the treatment of Type 2 Diabetes [11].

Although the potential risk of cancer associated with DPP 4 inhibitors has been widely discussed in the social media, the potential role of DPP 4 in tumor transformation and cancer development remains largely undefined [12]. Cheng and colleagues demonstrated DPP 4 involvement in the metastasis of rat breast cancer cells [13]. In a retrospective cohort study, Garg et al., demonstrated increased incidence of acute pancreatitis in diabetic patients, but failed to associate the increased incidence with the use of sitagliptin [14]. Based on the nested case-control study using the World Health Organization-Adverse Drug Reactions (WHO-ADR) database, Willemen et al., demonstrated increased events of infection, especially upper respiratory infections, associated with DPP 4 inhibitors [15]. Using the ADRs associated with sitagliptin reported to the FAERS from 2007 to 2009, Elashoff et al. demonstrated an increased risk of pancreatitis and pancreatic cancer associated with sitagliptin [16]. Pharmaceutical companies, however, rebuked the study results and generated a significant discussion in the social media and in the field of biomedical and clinical research [17].

The objective of this study is to assess the risk of pancreatic cancer associated with anti-diabetic drugs of dipeptidyl peptidase 4 (DPP 4) inhibitors with or without combination of metformin. These findings may provide future directions for clinical studies of cancer control and prevention and may have substantial impact on patient care, drug safety, cancer control and prevention in the changing media landscape and new information ecology.

\section{Methods}

\section{Setting and study design}

Data were obtained from the FAERS which contains 3937780 spontaneous adverse event case reports filed from 2007 to 2012 by the consumers, health care providers and pharmaceutical manufacturers regarding identifiable, but anonymous, patients. Each patient was assigned a unique ID by the reporting system. The FDA might identify the patients, but for the public, the patients are anonymous. The new cases of suspected adverse events are released to the public every quarter and the reports include 7 data sets: patient demographic and administrative information; drug information; adverse events; patient outcomes; report sources; drug therapy start and end dates; and indications for use [18].

This study was designed as a nested case-control study to analyze the role of the FAERS database in the evolving media landscape to delineate the cancer risks associated with DPP4 inhibitors. A Venn diagram of public health media landscape was constructed with a collection of major symbolic elements for each of the major media categories (Figure 1). In addition, the communication and information flow of drug adverse events among all the major elements of the public health media landscape were analyzed. The base cohort consisted of all ADRs associated with the use of sitagliptin and saxagliptin. The data

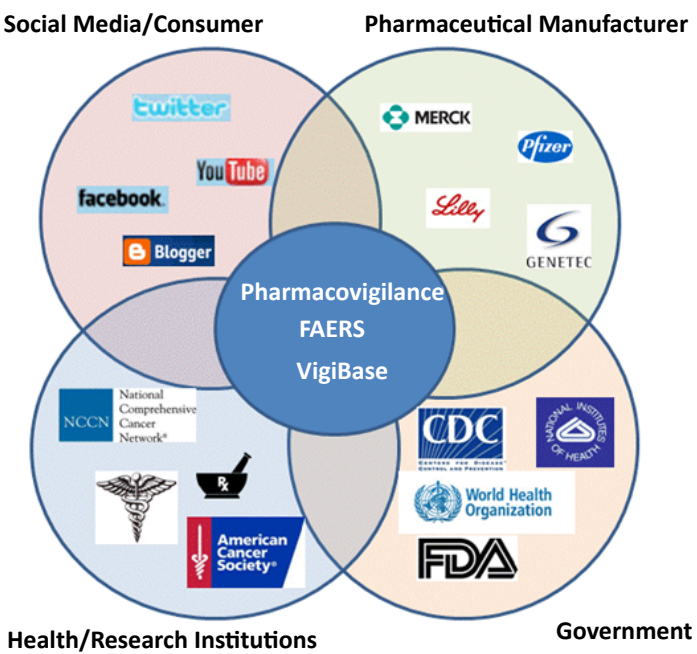

Figure 1: Venn diagram of the media landscape for the public health: This diagram clearly links today's evolving media landscape including traditional media, social media, pharmaceutical manufacturers, and government. The pharmacovigilance programs are usually sponsored by the government and national or international coalitions. The FAERS and the VigiBase, two most successful pharmacovigilance programs, are in the center of the media landscape to engage all the stakeholders of public health inmatters of drug safety, diseases control and prevention. FAERS: The U.S. FDA Adverse Events Reporting System. VigiBase: The World Health Organization (WHO) Individual Case Safety Reports Database System.

collection period ran from year 2007 when adverse drug event reports first became available for sitagliptin (approved by the FDA in 2006) to the ended of the first quarter of 2012. The controls were the ADRs associated with the rest of the drugs reported in the FAERS excluding DPP 4 inhibitors. A standard pharmacovigilance tool was used to identify the risk of cancer associated with the drug class of DPP 4 inhibitors $[19,20]$.

\section{Data processing}

Relational databases were first created from the ASCII files in the FAERS using Microsoft Office Access. Then the data were sorted and analyzed with Microsoft Excel analytic tools. The predefined events of interest were pancreatic cancer and other cancers. Using the FAERS public database, the ADRs associated with DPP 4 inhibitors were generated and evaluated. The most prevalent cancer signals were also identified. The ADRs involving combination drugs were not included in this study. Based on the ADRs from 2007 to 2012, the reported cancer adverse events associated with DPP 4 inhibitors were analyzed for sitagliptin (approved in October 2006) and saxagliptin (approved in July 2009). Linagliptin (approved in May 2011) was excluded from this study because of the insufficient number of adverse events.

\section{Statistical analysis}

Spontaneous ADRs associated with DPP 4 inhibitors were acquired from the FAERS database and analyzed using proportional reporting ratio (PRR) and reporting odds ratio (ROR) for signal generation from spontaneous adverse drug reaction reports. To detect risk signals with alarming reporting frequencies, PRR and ROR were calculated on the basis of all adverse events associated with all drugs reported in the FAERS from 2007 to 2012. Two by two contingency tables for 
Citation: Feng X, Cai A, Dong K, Chaing W, Feng M, et al. (2013) Assessing Pancreatic Cancer Risk Associated with Dipeptidyl Peptidase 4 Inhibitors: Data Mining of FDA Adverse Event Reporting System (FAERS). J Pharmacovigilance 1: 110. doi:10.4172/2329-6887.1000110

cancer events associated with or without the presence of sitagliptin or saxagliptin were created to calculate PRR and ROR including a two sided $95 \%$ confidence interval $(95 \% \mathrm{CI})$. A signal for alarm was considered for a PRR $>2$ or ROR $>2$, and if the lower bound of $95 \% \mathrm{CI}$ for ROR was greater than 1 . A linear regression analysis was performed to evaluate the correlation between the number of cancer events and time in years to evaluate the trend of cancer adverse events associated with drug therapy [21-25].

\section{Results}

\section{Pharmacovigilance and the media landscape of public health}

Drug safety is one of the most important issues for public health. The media landscape provides an essential platform for the public health community to communicate the information of potential premarket and post-market ADRs using digital social media, mobile text messaging, online chat and blogs, news stories in newspapers and magazines, case reports in research articles, official warnings and reports from organizations and federal agencies. The Venn diagram shown in Figure 1 provides a bird's eye view of the media landscape for the public health. The overwhelming drug safety information in the traditional media and the social media needs to be filtered and managed by the pharmacovigilance programs, which are usually sponsored by pharmaceutical companies, the government or national and international organizations. Thus Pharmacovigilance programs, such as the FAERS, are in the center of the evolving media landscape to engage all the stakeholders of public health for drug safety, diseases control and prevention.

Today's media landscape is facing a significant challenge of reporting the ADRs, such as cancer risks associated with drug treatments, due to privacy and liability issues. There is an urgent need to establish and expand the sophisticated pharmacovigilance programs at the government and international coalition level. The FAERS is one of the largest government administrated database information systems of spontaneous ADRs. It serves as a central hub of information flow for reporting ADRs in the changing media landscape and new information ecology (Figure 2). The early phase communication for suspected ADRs usually occurs among health professionals, individual consumers, and manufacturers. Then an identified potential adverse event associated with an identified drug product for an identifiable, but anonymous, patient can be reported to the FAERS.

Although, this database with millions of ADRs is available to the

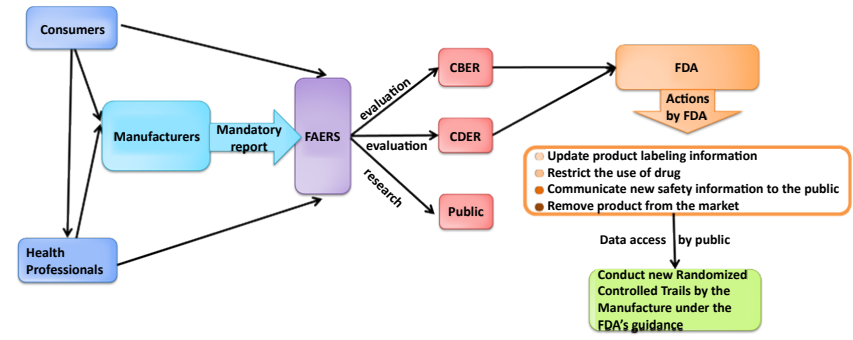

Figure 2: Pathways of FAERS work flow: The FAERS serves as a central hub for information flow of adverse events report. The early phase communication for suspected ADRs usually occurs among health professionals, individual consumers, and manufacturers. Then, the data are shared and evaluated by the Center for Biologics Evaluation and Research (CBER), Center for Drug Evaluation and Research (CDER), and the public. Eventually, the recommendations and actions from the FDA, which lead to the public awareness are broadcasted to the public. public, the FAERS is often unsearchable and incomprehensible for the general public consumers. On the other hand, the FAERS provides an essential platform for the clinicians, biomedical researchers, the pharmaceutical companies, the federal agencies, and other health care stakeholders to study the data and identify alarming signals, such as cancer risks associated with drugs. The most significant and newly identified post-market adverse events are assessed, evaluated and confirmed by well designed clinical trials. Based on the FAERS assessment and clinical evidence, the government communicates with the consumers, the health care providers and the manufacturers, and might even uses its authority to regulate the suspected drugs (Figure 2).

Based on its pharmacovigilance assessment, recently the FDA issued a safety announcement for popular anti-diabetic drug pioglitazone to inform the public that use of pioglitazone for more than one year may be associated with an increased risk of bladder cancer. Our data indicated that the bladder cancer cases associated with pioglitazone reported in the FAERS increased 40 times after the FDA safety announcement (data not shown). This is a perfect example of using pharmacovigilance data to identify cancer risks, enhance cancer risk awareness, and promote cancer control and prevention in the new information ecology.

\section{Increasing trend of consumer reports and pancreatic cancer cases in the FAERS}

The government should expand the role of the FAERS in cancer control and prevention. Unlike other health care databases which collect information mainly from health care providers, the FAERS encourages individual consumers to file ADRs to the FAERS directly. In 2010, the consumers filed 403,746 adverse events in the FAERS and the health care providers filed 427,064 adverse events. The total number of FAERS cases contributed by individual consumers increased over 10 times in the past 10 years. Consumer is the biggest contributing group for the FAERS cases with fastest growth trend compared to physicians, pharmacists and other healthcare providers In contrast; pharmacist is the contributing group with least contributions and least growth (Figure 3 ). In addition, the number of pancreatic cancer adverse events associated with drug use reported in the FAERS has been escalating from 2006 to 2011. The total incidence of pancreatic cancer adverse events filed in the FAERS was tripled in 2011 ( 781 cases) compared to that of 2006 ( 270 cases) (Table 1).

\section{Association of cancer risks with DPP 4 inhibitors}

A total of 12,618 ADRs associated with sitagliptin were reported to the FAERS from 2007 to 2011. Although the total number ADRs vary over time, there was no evidence of an upward trend. During the same time period, a total of 223 cancer events associated with sitagliptin were reported to the FAERS. However, unlike the data for total ADRs, there was a noticeable upward trend in sitagliptin related cancers (Figure 4A). Linear regression analysis indicated there was a significant correlation between the proportional reporting ratio and time $(R=0.796 ; p<0.001)$ for sitagliptin. Since the FAERS database was published quarterly, quarter was used on $\mathrm{X}$-axis (Figure $4 \mathrm{~B}$ ).

By the end of 2011, there were 14 types of suspected malignant events associated with sitagliptin reported to the FAERS including: pancreatic cancer, leukemia, lung cancer, breast cancer, bladder cancer, lymphoma, hepatic cancer, skin cancer, colorectal cancer, ovarian cancer, endometrial cancer, prostate cancer, thyroid cancer and myeloma. The five most prevalent cancer events associated with sitagliptin were: pancreatic cancer (49 cases), leukemia ( 28 cases), lung cancer (20 cases), breast cancer (16 cases) and bladder cancer (14 cases). Pancreatic cancer accounted for $22 \%$ of all combined cancer events (Figure $4 \mathrm{C}$ ). 


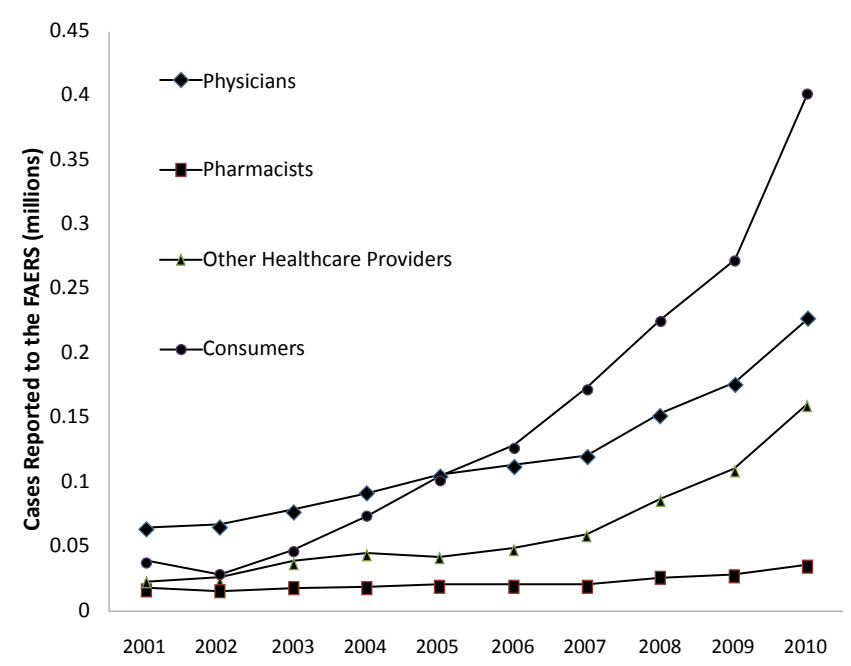

Figure 3: Growth trend of the FAERS reports contributed by the consumers and health care providers: The data were obtained from the FAERS from 2001 to 2010 and the growth trend for reports contributed by consumers, physicians, pharmacists, and other healthcare was analyzed. The reports contributed by the consumers have the biggest growth compared to those of physicians, pharmacists, and other health care providers [6].

Saxagliptin was the second DPP 4 inhibitor approved by the FDA in July 2009 to treat type 2 Diabetes. There were a total of 1,656 adverse events associated with saxagliptin reported to the FAERS from 2009 to 2011, including 35 cases of cancers. No cancer events associated with saxagliptin were reported in 2009, 11 cancer cases were reported in 2010 and 24 cases were reported in 2011. By the end of 2011, there were 9 types of malignancy events associated with using saxagliptin reported to the FAERS, which included: leukemia, pancreatic cancer, bladder cancer, hepatic cancer, skin cancer, lymphoma, prostate cancer, thyroid cancer and rhabodomyasarcoma. As with sitagliptin, there were over $20 \%$ of the total cancer adverse events associated with saxagliptin were pancreatic cancer (data not shown). The third DPP 4 inhibitor, linagliptin, was approved by the FDA for type 2 Diabetes in May 2011 and there were not enough data in the FAERS for analysis by the end of 2011. Thus pancreatic cancer was one the most commonly reported cancer adverse events associated with DPP 4 inhibitors in FAERS

\section{Metformin decreased pancreatic cancer risk Associated with DPP 4 inhibitors}

The algorithm of pharmacovigilance was used for data mining from the FAERS to assess the signals of pancreatic cancer adverse events associated with sitagliptin, saxagliptin and the drug class of DPP 4 inhibitors (Table 2). The signal of pancreatic cancer adverse events associated with sitagliptin was substantial and statistically significant ( $\mathrm{PRR}=6.07$; and ROR=6.139; 95\% CI: $5.152-7.316$; $\mathrm{P}<0.0005)$. The control was all pancreatic cancer cases associated with all drugs in the FERS excluding sitagliptin. Similar substantial and statistically significant pharmacovigilance signal was detected for saxagliptin ( $P R R=4.54$, and $\mathrm{ROR}=4.551 ; 95 \% \mathrm{CI}: 2.961-6.996 ; \mathrm{P}<0.0005)$ and $\mathrm{DPP}$ 4 inhibitors drug class $(\mathrm{PRR}=5.90$, and $\mathrm{ROR}=5.922$; $95 \% \mathrm{CI}$ : 5.029 6.972; $\mathrm{P}<0.0005)$.

Interestingly, significantly less adverse events of pancreatic cancer associated with treatment of metformin were reported to the AERS (Table 3). The risk signal of pancreatic cancer associated with metformin was not significant ( $P R R=1.21)$ compared to that of sitegliptin $(\mathrm{PRR}=6.07)$. Furthermore, the risk of pancreatic cancer associated with sitagliptin was decreased $72.3 \%$ by using the combination of metformin with sitagliptip compared to sitagliptin monotherapy (OR=0.277, 95\%: 0.210-0.365).

\section{Discussion}

Early detection and management of cancer risks associated with pharmacotherapy has been an essential approach for cancer control and cancer prevention. For example, Chlebowski et al., demonstrated in the Women's Health Initiative (WHI) trial that the rate of death from breast cancer among those taking estrogen plus progestin was twice of those taking the placebo [26]. However, the efforts of early detection of cancer risks associated with pharmacotherapy have been hindered by lack of powerful and sophisticated systems that integrate the everexpanding media landscape with health care information database, such as spontaneous reporting databases for adverse events [27].

The FAERS database, as a tool for pharmacovigilance programs, offers a significant opportunity for the general public as well as for the health care professionals to discuss, inquire, and report health care information. Based on the FAERS database, over 100 research papers have been published to assess the post-market risks of adverse events associated with health care products [28-30]. In this report, we demonstrate that the frequency of drug related pancreatic cancers reported to the FAERS has nearly tripled from 270 in year 2006 to 781 in year 2011. Using pharmacovigilance algorithms we demonstrated that there was a significant high rate of cancer events correlated with sitagliptin use, the first DPP4 inhibitor approved by the FDA in October 2006. This increasing trend of correlation could due to longer exposure or more sitegliptin are prescribed. According to IMS Health, the sale of sitagliptin in US has been steadily increasing (billion dollars: 0.6 in 2007, 1.2 in 2008, 1.5 in 2009, 1.7 in 2010, and 2.1 in 2011) [31] The trend was similar for saxagliptin, the second DPP4 inhibitor drug approved by the FDA for type 2 diabetes. The results from this study indicated that there was a significant association of pancreatic cancer risks associated with using sitagliptin, saxagliptin and DPP4 inhibitor drug class.

These results are consistent with the previous published pharmacovigilance study published by Elashoff et al., which assessed the FAERS reports of adverse events associated with sitagliptin from 2007 to 2009 [16]. Our extensive study indicated the pancreatic cancer risk could be a class effect for DPP 4 inhibitors. It is well known that pancreatitis is a potential etiologic risk for pancreatic cancer [32]. After a thorough analysis of 88 FAERS cases of acute pancreatitis in patients using sitagliptin or sitagliptin combinations from 2006 to 2009, the FDA now included the association of pancreatitis with sitagliptin in the prescribing information [33]. Furthermore, DPP 4 has multiple functions in cleaving a variety of physiologically and pathophysiologically important peptides in the circulation, such as incretin hormones glucagon-like peptide-1 (GLP-1) and glucosedependent insulinotropic polypeptide (GIP), certain chemokines, and mitogenic growth factors, which might involved in tumor transformation and cancer development [34].

New evidence indicated that type 2 diabetic patient have higher risk of cancer development and cancer mortality. Yang and colleagues demonstrated that In Chinese patients with type 2 diabetes, there is a significant correlation between Alc levels and cancer occurrences. 
Citation: Feng X, Cai A, Dong K, Chaing W, Feng M, et al. (2013) Assessing Pancreatic Cancer Risk Associated with Dipeptidyl Peptidase 4 Inhibitors: Data Mining of FDA Adverse Event Reporting System (FAERS). J Pharmacovigilance 1: 110. doi:10.4172/2329-6887.1000110

Page 5 of 7

\begin{tabular}{|c|c|c|c|c|c|}
\hline & $\begin{array}{c}\text { Q1: Total ADRs } \\
\text { (pancreatic Cancer) }\end{array}$ & $\begin{array}{c}\text { Q2: Total ADRs (pancreatic } \\
\text { Cancer) }\end{array}$ & $\begin{array}{c}\text { Q3: Total ADRs } \\
\text { (pancreatic Cancer) }\end{array}$ & $\begin{array}{c}\text { Q4: Total ADRs } \\
\text { (pancreatic Cancer) }\end{array}$ & $\begin{array}{c}\text { Annual Total ADRs (Annual } \\
\text { pancreatic Cancer) }\end{array}$ \\
\hline 2006 & $89,527(73)$ & $79,597(74)$ & $71,724(62)$ & $83,229(61)$ & $324,077(270)$ \\
\hline 2007 & $88,832(62)$ & $83,977(64)$ & $96,035(73)$ & $109,892(62)$ & $378,736(261)$ \\
\hline 2008 & $104,412(62)$ & $107,503(72)$ & $108,453(111)$ & $121,024(117)$ & $441,392(362)$ \\
\hline 2009 & $111,438(104)$ & $112,481(129)$ & $130,072(144)$ & 137,708 (169) & $491,699(546)$ \\
\hline 2010 & $136,191(151)$ & $144,452(136)$ & 209,307 (159) & $183,567(161)$ & $673,517(607)$ \\
\hline 2011 & $181,140(161)$ & $194,310(231)$ & 198,777 (199) & $208,742(196)$ & $782,969(781)$ \\
\hline 2012 & $835390(277)$ & & & & \\
\hline
\end{tabular}

ADRs: adverse event reports, FAERS: Food and Drug Administration Adverse Events Reporting System

Table 1: Quarterly and annual cases of pancreatic cancer and total ADRs filed in the FAERS from the first quarter of 2006 to the first quarter of 2012.
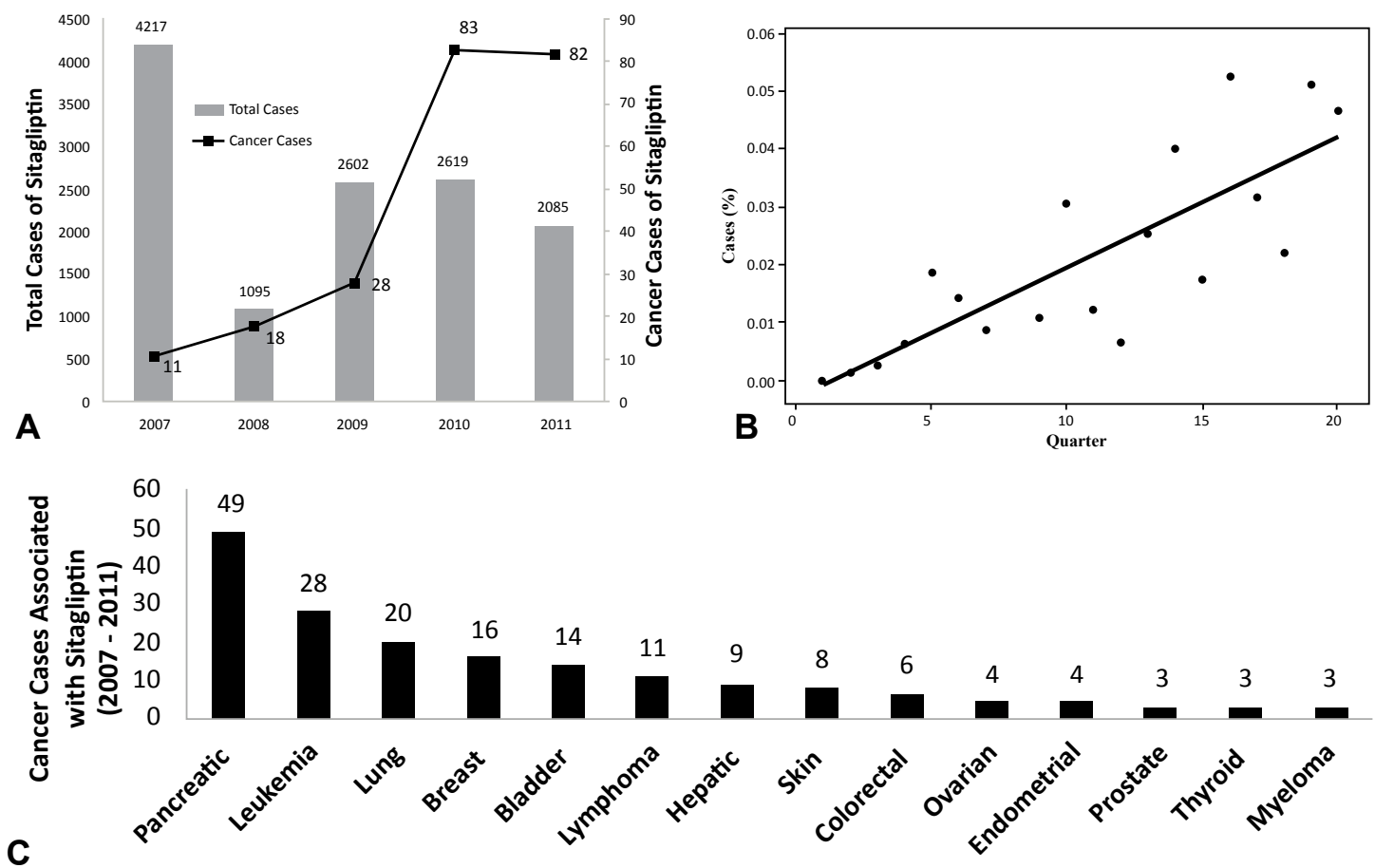

Figure 4: Association of Cancer Risks with sitagliptin in the FAERS from 2007 to 2011. The total cases of cancer adverse events and total cases of all adverse events associated with sitagliptin were calculated (A). Linear regression analysis indicated there was a significant correlation between the proportional reporting ratio (cancer cases over total cases) and time $(R=0.796 ; p<0.001)$ for sitagliptin $(B)$. By the end of 2011 , there were 14 types of suspected malignant events associated with sitagliptin reported to the FAERS $(\mathrm{C})$.

Their evidence also indicates that there is decreasing cancer risk associated with insulin usage [35]. In contrast, we demonstrated that DPP 4 inhibitor treatment correlates with substantially higher rate of pancreatic cancer in type 2 diabetic patients.

For the first time we demonstrated that combination of metformin with DPP 4 inhibitors correlates with significantly lower rate of pancreatic cancer compared to the treatment of DPP 4 inhibitor without matformin in FAERS. It suggests that treatment with metformin might correlate with lower rate of pancreatic cancer in patients with type 2 diabetes. In a large population-based follow-up study, Ruiter and colleagues demonstrated that use of metformin is associated with a lower risk of cancer compared with use of sulfonylurea derivatives, especially for pancreatic cancer [36]. Moreover, Kisfalvi et al. demonstrated in vivo evidence that metformin inhibites the growth of pancreatic cancer xenografts inbude mice [37]. Our study thus indicated that combination of metformin with DPP 4 inhibitors has potential benefit beyond glycemia control. Pharmacovigilance program like the FAERS will accelerate the effort of identifying cancer risks, enhancing cancer risk awareness, and promoting cancer control and prevention in the new information ecology. Millions of adverse events documented in the FAERS database provide valuable resources for post-market pharmacovigilance assessment for cancer control and prevention $[38,39]$.

The FAERS has been evolving as an essential component of today's revolutionary digital media landscape for public health by inquiring, sharing and reporting drug adverse events at manylevels using traditional and innovative media tools. Unlike other health care databases which mainly collect information from health care providers, the FAERS encourages individual consumers to file adverse event reports to the FAERS directly. In 2010 the consumers filed 403.746 adverse events in 
Citation: Feng X, Cai A, Dong K, Chaing W, Feng M, et al. (2013) Assessing Pancreatic Cancer Risk Associated with Dipeptidyl Peptidase 4 Inhibitors: Data Mining of FDA Adverse Event Reporting System (FAERS). J Pharmacovigilance 1: 110. doi:10.4172/2329-6887.1000110

\begin{tabular}{|c|c|c|c|c|c|c|}
\hline \multirow[b]{2}{*}{ Exposure } & \multicolumn{2}{|c|}{$\begin{array}{c}\text { Sitagliptin (2007-2012) } \\
3937780(3104)\end{array}$} & \multicolumn{2}{|c|}{$\begin{array}{l}\text { Saxagliptin } \\
(2009-2012)\end{array}$} & \multicolumn{2}{|c|}{$\begin{array}{l}\text { DPP } 4 \text { inhibitors } \\
(2007-2012)\end{array}$} \\
\hline & Yes & No & Yes & No & Yes & No \\
\hline Cases & 131 & 2803 & 21 & 5880 & 152 & 33919 \\
\hline Controls 1 & 297 & 3900 & 308 & 3928796 & 295 & 3900757 \\
\hline ROR & \multicolumn{2}{|c|}{$\begin{array}{l}\text { ROR=6.139 } \\
\text { 95\% Cl: } 5.152-7.316 \\
\text { P<0.0005 }\end{array}$} & \multicolumn{2}{|c|}{$\begin{array}{l}\text { ROR }=4.551 \\
95 \% \mathrm{Cl}: 2.961-6.996 \\
\mathrm{P}<0.0005\end{array}$} & \multicolumn{2}{|c|}{$\begin{array}{l}\mathrm{ROR}=5.922 \\
95 \% \mathrm{Cl}: \quad 5.029-6.972 \\
\mathrm{P}<0.0005\end{array}$} \\
\hline RR & \multicolumn{2}{|l|}{6.07} & \multicolumn{2}{|l|}{4.54} & \multicolumn{2}{|l|}{5.90} \\
\hline
\end{tabular}

DPP4: dipeptidyl peptidase 4, FAERS: Food and Drug Administration Adverse Events Reporting System, ROR: the reporting odds ratio (ROR), PRR: the proportional reporting ratio

Table 2: Pharmacovigilance assessment of pancreatic cancer risk signals associated with DPP 4 inhibitor drugs reported in FAERS from 2007 to 2012.

\begin{tabular}{|c|c|c|c|c|c|c|}
\hline \multirow[b]{2}{*}{ Exposure } & \multicolumn{2}{|c|}{$\begin{array}{l}\text { Sitagliptin } \\
(2007-2012)\end{array}$} & \multicolumn{2}{|c|}{$\begin{array}{c}\text { metformin } \\
(2004-2012)\end{array}$} & \multicolumn{2}{|c|}{$\begin{array}{c}\text { Sitagliptin+metformin } \\
(2007-2012)\end{array}$} \\
\hline & Yes & No & Yes & No & Yes & No \\
\hline Cases & 131 & 28039 & 49 & 51289 & 83 & 64167 \\
\hline Controls 1 & 2973 & 3906637 & 3055 & 3883387 & 3021 & 3870509 \\
\hline ROR & \multicolumn{2}{|c|}{$\begin{array}{l}\mathrm{ROR}=6.139 \\
95 \% \mathrm{Cl}: 5.152-7.316 \\
\mathrm{P}<0.0005\end{array}$} & \multicolumn{2}{|c|}{$\begin{array}{l}\mathrm{ROR}=1.214 \\
95 \% \mathrm{Cl}: 0.916-1.611 \\
\mathrm{P}=0.178\end{array}$} & $\begin{array}{l}\text { ROR } \\
95 \% \mathrm{C} \\
\mathrm{P}<0.0\end{array}$ & $1.332-2.061$ \\
\hline PRR & 6.07 & & 1.21 & & 1.66 & \\
\hline
\end{tabular}

FAERS: Food and Drug Administration Adverse Events Reporting System, ROR: the reporting odds ratio (ROR), PRR: the proportional reporting ratio

Table 3: Metformin significantly decreased pancreatic cancer risk associated with sitagliptin reported in FAERS from 2007 to 2012.

the FAERS and the health providers filed 427,064 adverse events. The total number of AERS cases contributed by consumers increased over ten times during the last decade [40]. The success of public involvement in the FAERS can be translated to other public databases of heath care. Nevertheless, our data also indicated that pharmacists' contribution to the FAERS should be improved. The cases contributed to the FAERS by the pharmacist was not even doubled in the past decade (19050 cases in 2001, 36448 cases in 2010) In addition, the total cases in the FAERS contributed by the pharmacists in 2010 was only $9 \%$ of total cases contributed by the individual consumers (36448 cases versus 403746 cases) and $8.5 \%$ of total cases contributed by all healthcare providers.

The major limitation of the FAERS is potentially biased reporting: under reporting for rare unpublished adverse events and potential over reporting for published adverse events. There is an urgent need to develop social or mobile tools to encourage consumers and health care professionals to file adverse event report to the FAERS $[3,41]$. In addition, the information in the FAERS can be incomprehensible and unsearchable for most consumers. Since the case reporting for the FAERS is spontaneous, there are potential limitations of reporting biases, incomplete reporting, under reporting, and Webereffect [27]. Although randomized, controlled clinical trials remain the gold standard to assess pre-market and post-market drug safety; pharmacovigilance systems like the FAERS plays a critical role to raise the clinical and public awareness of cancer risks, and to direct future longitudinal studies for cancer control and prevention in the changing media landscape and new information ecology.

\section{References}

1. Apostolakis I, Koulierakis G, Berler A, Chryssanthou A, Varlamis I (2012) Use of social media by healthcare professionals in Greece: an exploratory study. Int J Electron Healthc 7: 105-124.

2. Vandewater EA, Denis LM (2011) Media, social networking, and pediatric obesity. Pediatr Clin North Am 58: 1509-1519, xii.
3. Chou WY, Hunt YM, Beckjord EB, Moser RP, Hesse BW (2009) Social media use in the United States: implications for health communication. J Med Internet Res 11: e48.

4. Banerjee AK, Ingate S (2012) Web-based patient-reported outcomes in drug safety and risk management: challenges and opportunities? Drug Saf 35: 437446.

5. Borg JJ, Aislaitner G, Pirozynski M, Mifsud S (2011) Strengthening and rationalizing pharmacovigilance in the EU: where is Europe heading to? A review of the new EU legislation on pharmacovigilance. Drug Saf 34: 187-197.

6. FDA Adverse Event Reporting System (FAERS), FDA, U.S. Department of Health and Human Services, USA.

7. Hoffman KB, Kraus C, Dimbil M, Golomb BA (2012) A survey of the FDA's AERS database regarding muscle and tendon adverse events linked to the statin drug class. PLoS One 7: e42866.

8. Piccinni C, Motola D, Marchesini G, Poluzzi E (2011) Assessing the association of pioglitazone use and bladder cancer through drug adverse event reporting. Diabetes Care 34: 1369-1371.

9. Hoover R, Fraumeni JF Jr (1981) Drug-induced cancer. Cancer 47:1071-1080.

10. Stolley PD, Zahm SH (1995) Nonhormonal drugs and cancer. Environ Health Perspect 103 Suppl 8: 191-196.

11. Aroda VR, Henry RR, Han J, Huang W, DeYoung MB, et al. (2012) Efficacy of GLP-1 receptor agonists and DPP-4 inhibitors: meta-analysis and systematic review. Clin Ther 34: 1247-1258.

12. Giovannucci E, Harlan DM, Archer MC, Bergenstal RM, Gapstur SM, et al (2010) Diabetes and cancer: a consensus report. Diabetes Care 33: 16741685

13. Cheng HC, Abdel-Ghany M, Elble RC, Pauli BU (1998) Lung endothelial dipeptidyl peptidase IV promotes adhesion and metastasis of rat breast cancer cells via tumor cell surface-associated fibronectin. J Biol Chem 273: $24207-$ 24215.

14. Garg R, Chen W, Pendergrass M (2010) Acute pancreatitis in type 2 diabetes treated with exenatide or sitagliptin: a retrospective observational pharmacy claims analysis. Diabetes Care 33: 2349-2354.

15. Willemen MJ, Mantel-Teeuwisse AK, Straus SM, Meyboom RH, Egberts TC, et al. (2011) Use of dipeptidyl peptidase-4 inhibitors and the reporting of infections: a disproportionality analysis in the World Health Organization VigiBase. Diabetes Care 34: 369-374

16. Elashoff M, Matveyenko AV, Gier B, Elashoff R, Butler PC (2011) Pancreatitis pancreatic, and thyroid cancer with glucagon-like peptide-1-based therapies Gastroenterology 141: 150-156.

17. Hawkes N (2011) Journal withdraws article after complaints from drug manufacturers. BMJ 342: d2335.

18. U.S. Food and Drug Administration (FDA) Adverse Event Reporting System (AERS), USA.

19. Bate A, Evans SJ (2009) Quantitative signal detection using spontaneous ADR reporting. Pharmacoepidemiol Drug Saf 18: 427-436.

20. Gould AL (2003) Practical pharmacovigilance analysis strategies Pharmacoepidemiol Drug Saf 12: 559-574.

21. Almenoff JS, Pattishall EN, Gibbs TG, DuMouchel W, Evans SJ, et al. (2007) Novel statistical tools for monitoring the safety of marketed drugs. Clin Pharmacol Ther 82: 157-166.

22. Evans SJ, Waller PC, Davis S (2001) Use of proportional reporting ratios (PRRs) for signal generation from spontaneous adverse drug reaction reports. Pharmacoepidemiol Drug Saf 10: 483-486.

23. van Puijenbroek EP, Bate A, Leufkens HG, Lindquist M, Orre R, et al. (2002) A comparison of measures of disproportionality for signal detection in spontaneous reporting systems for adverse drug reactions. Pharmacoepidemiol Drug Saf 11: 3-10.

24. Bate A, Lindquist M, Edwards IR, Olsson S, Orre R, et al. (1998) A Bayesian neural network method for adverse drug reaction signal generation. Eur $\mathrm{J}$ Clin Pharmacol 54: 315-321.

25. Szarfman A, Machado SG, O'Neill RT (2002) Use of screening algorithms and computer systems to efficiently signal higher-than-expected combinations of 
Citation: Feng X, Cai A, Dong K, Chaing W, Feng M, et al. (2013) Assessing Pancreatic Cancer Risk Associated with Dipeptidyl Peptidase 4 Inhibitors: Data Mining of FDA Adverse Event Reporting System (FAERS). J Pharmacovigilance 1: 110. doi:10.4172/2329-6887.1000110

drugs and events in the US FDA's spontaneous reports database. Drug Saf. 25: 381-392.

26. Chlebowski RT, Anderson GL, Gass M, Lane DS, Aragaki AK, et al. (2010) Estrogen plus progestin and breast cancer incidence and mortality in postmenopausal women. JAMA 304: 1684-1692.

27. Hochberg AM, Pearson RK, O'Hara DJ, Reisinger SJ (2009) Drug-versus-drug adverse event rate comparisons: a pilot study based on data from the US FDA Adverse Event Reporting System. Drug Saf 32: 137-146.

28. Sakaeda T, Kadoyama K, Okuno Y (2011) Adverse event profiles of platinum agents: data mining of the public version of the FDA adverse event reporting system, AERS, and reproducibility of clinical observations. Int. J. Med. Sci. 8: 487-491.

29. Sakaeda T, Kadoyama K, Yabuuchi H, Niijima S, Seki K, et al. (2011) Platinum agent-induced hypersensitivity reactions: data mining of the public version of the FDA adverse event reporting system, AERS. Int J Med Sci 8: 332-338.

30. Kadoyama K, Kuwahara A, Yamamori M, Brown JB, Sakaeda T, et al. (2011) Hypersensitivity reactions to anticancer agents: data mining of the public version of the FDA adverse event reporting system, AERS. J Exp Clin Cancer Res 30: 93.

31. IMS Health. Top U.S Pharmaceutical Products by Spending.

32. Hart AR, Kennedy H, Harvey I (2008) Pancreatic cancer: a review of the evidence on causation. Clin Gastroenterol Hepatol 6: 275-282.

33. Information for Healthcare Professionals - Acute pancreatitis and sitagliptin (marketed as Januvia and Janumet) [09-25-2009], FDA, USA
34. Ghersi G, Zhao Q, Salamone M, Yeh Y, Zucker S, et al. (2006) The protease complex consisting of dipeptidyl peptidase IV and seprase plays a role in the migration and invasion of human endothelial cells in collagenous matrices. Cancer Res 66: 4652-4661.

35. Yang X, Ko GT, So WY, Ma RC, Yu LW, et al. (2010) Associations of hyperglycemia and insulin usage with the risk of cancer in type 2 diabetes: the Hong Kong diabetes registry. Diabetes 59: 1254-1260.

36. Ruiter R, Visser LE, van Herk-Sukel MP, Coebergh JW, Haak HR, et al. (2012) Lower risk of cancer in patients on metformin in comparison with those on sulfonylurea derivatives: results from a large population-based follow-up study. Diabetes Care 35: 119-124.

37. Kisfalvi K, Moro A, Sinnett-Smith J, Eibl G, Rozengurt E (2013) Metformin inhibits the growth of human pancreatic cancer xenografts. Pancreas 42: 781785

38. FDA Drug Safety Communication: Update to ongoing safety review of Actos (pioglitazone) and increased risk of bladder cancer [6-15-2011], FDA, USA.

39. Mosholder AD, Pamer CA (2006) Postmarketing surveillance of suicidal adverse events with pediatric use of antidepressants. J Child Adolesc Psychopharmacol 16: 33-36.

40. AERS Reporting by Healthcare Providers and Consumers by Year (As of December 31, 2010).

41. Curioso WH, Karras BT, Campos PE, Buendia C, Holmes KK, et al. (2005) Design and implementation of Cell-PREVEN: a real-time surveillance system for adverse events using cell phones in Peru. AMIA Annu Symp Proc . 